\title{
Gene Regulatory Networks Governing the Generation and Regeneration of Blood
}

\author{
ALDO CIAU-UITZ and ROGER PATIENT
}

\begin{abstract}
Blood is an example of a highly regenerative tissue and its regeneration depends on the presence of stem cells residing in the bone marrow in humans. A better understanding of how these stem cells are programmed would benefit their use in clinical practice and shed light on the mechanisms by which the unique properties of stem cells are established. Our approach is to delineate the gene regulatory networks (GRNs) that specify these cells during their development in the embryo, and we use the amphibian experimental model because a wealth of evidence shows that the mechanisms used are conserved in mammals including humans. Blood stem cells are made during the intraembryonic wave of hematopoiesis during embryonic development where they emerge from endothelial precursors in the floor of the dorsal aorta (DA). These cells are derived from lateral plate mesoderm and so we have focused on the subset of cells in the lateral plate mesoderm fated to become blood and endothelium known as definitive hemangioblasts. We have found that their programming results from the activities of vascular endothelial growth factor A (VEGFA) and bone morphogenetic protein (BMP) signaling and the inhibition by miRNA of transforming growth factor beta signaling. VEGFA is first generated in the somites adjacent to the lateral plate mesoderm, and one of the responses of the lateral plate mesoderm is to activate endogenous VEGFA expression. BMP has multiple inputs into the programming of these cells via the activation of the transcription factor (TF), Gata2, and of the VEGFA receptor. These actions culminate in the expression of the leukemia-associated TF, Scl/Tal1, which is essential for blood fate specification. The activity of VEGFA in driving endothelial development resides in the small isoform, but the medium and large isoforms are required to initiate the blood stem cell program in the floor of the DA. The expression of the small isoform is dependent on the blood TF with leukemia connections, Tel1/Etv6, whereas the larger isoforms depend on another transcription-associated factor with leukemia connections, Eto2, raising the possibility that the regulation of VEGFA expression may be the mode of action of these leukemic factors. The action of Tel1/Etv6 in directly activating VEGFA expression in the somites was unexpected because this TF had only been reported to repress transcription. Using chromatin immunoprecipitation technology, we were able to show that Tel1/Etv6 does indeed work by repressing the expression of a VEGFA repressor, FoxC3, but it also acts directly to activate VEGFA expression, working together with Klf4. Finally, we have also looked at the mesodermal population that gives rise to the earlier waves of hematopoiesis,
\end{abstract}

Weatherall Institute of Molecular Medicine, John Radcliffe Hospital, University of Oxford, Oxford, United Kingdom. 
which do not generate a stem cell. We find significant differences including differential use of TFs of the E-Twenty-Six (ETS) family. In conclusion, we have elucidated the GRN responsible for preparing the lateral mesoderm for blood stem cell production.

Keywords: blood, gene regulatory networks, stem cells.

$\mathbf{T}$

HE READING OF INFORMATION from the genome differs between cells, and therefore, the essence of cell

identity is the setting up of different gene expression programs. The expression of individual genes depends on proteins that bind DNA, recognizing specific DNA sequences, leading to the opening of local chromatin structure, and facilitating the binding of additional proteins, including eventually the RNA polymerase that will copy the DNA of the gene body into RNA and then to protein.

During the lifetime of vertebrate organisms, there is an ongoing requirement for the generation of new blood cells due to constant turnover. This process depends on a small population of stem cells in the adult, located in the bone marrow in mammals including humans. These cells are specified by their gene expression program to be blood, as opposed to other tissues; however, they retain the potential to make all the different cells of the blood, being so-called multipotent. The numbers of each blood cell type made are responsive to need and mediated by the environmental conditions in which the stem cells are located. The critical drivers of the selection of the separate programs are extracellular signaling molecules called cytokines, which determine which DNA-binding proteins are able to bind to the DNA and drive gene expression.

A critical cell in the generation of blood is therefore the blood or hematopoietic stem cell (HSC). As already stated, this cell is differentiated in that it can only make blood, but it still retains multipotency with respect to which blood cells are made. Therefore, an important aspect of understanding blood generation is to elucidate the development and programming of the HSC. Because HSCs have the capacity to regenerate the entire hematopoietic system upon transplantation, they play a key role in bone marrow and umbilical cord transplants performed to restore hematopoiesis in patients with blood diseases. However, insufficient numbers of appropriate donors and a poor understanding of the biology of human HSCs preclude their wider application in the clinic. Therefore, methods for amplification or de novo generation of HSCs from pluripotent stem cells (PSCs) are desirable. Unfortunately, despite intense efforts, the efficient generation of bona fide HSCs from PSCs has not been achieved so far (Ivanovs et al., 2017). The embryo efficiently generates and expands HSCs; therefore, the developmental biology of HSCs holds essential information for the establishment of protocols for the generation of HSCs in vitro for clinical use.

Blood cells of various types are needed in the developing embryo before HSCs are made. Indeed, there are at least three waves of blood formation or hematopoiesis during development (Yoder, 2014). The first wave takes place in the yolk sac of mammalian embryos and consists primarily of red blood cells critical for oxygenation of the growing tissues in the embryo. Some so-called myeloid cells, consisting of macrophages and neutrophils that are part of the innate immune system, are also made at this time. The second wave of hematopoiesis also occurs in the yolk sac and contains yet more different cell types, including lymphocytes of the acquired immune system, with the final wave taking place outside the yolk sac, in the embryo proper, giving rise to the HSCs and other more short-lived multipotent progenitors.

The site of HSC production in the developing embryo is the dorsal aorta (DA). Indeed, there is strong evidence that HSCs derive from the endothelial cells that make up the floor of the DA (Bertrand et al., 2010; Boisset et al., 2010; Kissa and Herbomel, 2010). Thus, a full understanding of HSC development and programming needs to include knowledge of the mechanisms by which the DA is specified.

By tracking the cells in the embryo, we showed nearly 20 years ago that the cells giving rise to the first two waves of hematopoiesis have distinct origins from those that make the DA and HSCs (Ciau-Uitz et al., 2000). The earliest mesodermal progenitors to HSCs (ventral lateral marginal zone, Fig. 1A) and the first two waves of hematopoiesis (dorsal marginal zone, Fig. 2A) are born in different locations and times during gastrulation. This was an important discovery because the cells take different paths through the embryo, and therefore, the blood programs in the different populations are influenced by distinct combinations of 

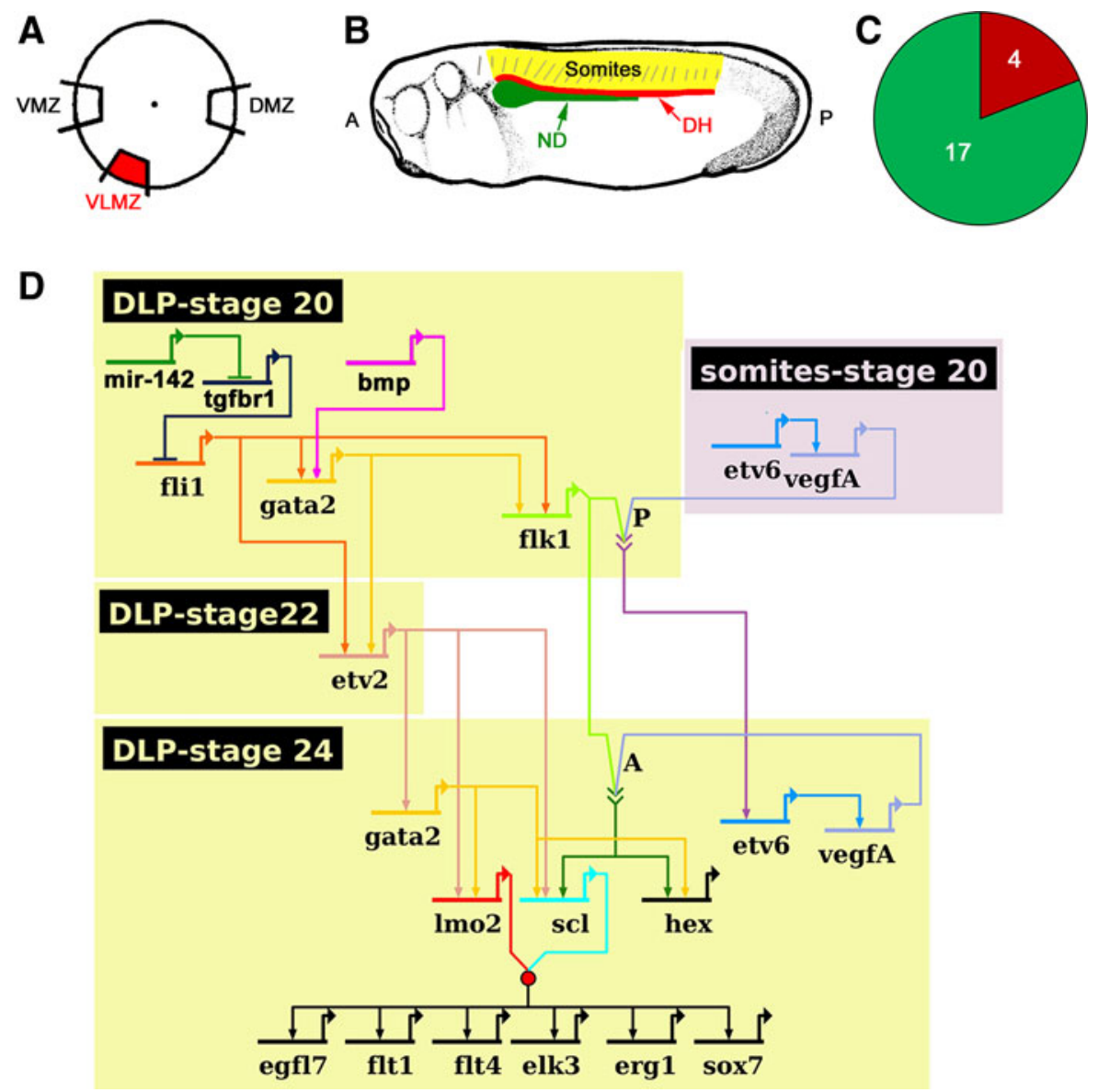

FIG. 1. Origins and programming of adult DH. (A) DH and HSCs derive from the VLMZ region of the gastrula embryo. (B) DHs emerge in close association with the somites and the ND during early organogenesis. (C) Pie chart illustrating the proportion of hematopoietic (red) and endothelial (green) gene expression in DHs. Numbers indicate the number of genes expressed in each category. (D) The DH gene regulatory network. DH, definitive hemangioblast; HSCs, hematopoietic stem cells; ND, nephric duct; VLMZ, ventral lateral marginal zone.

embryonic signals and thereby distinct DNA-binding proteins, known as transcription factors (TFs), are activated.

TFs are themselves the protein products of genes, and their expression is controlled at the level of transcription under the control of TFs. Therefore, one needs to understand the networks of TF gene expression that are set up in the different blood cells as they are being programmed. In this way, one would hope to build an understanding of why the blood cells of the first two waves of hematopoiesis develop into fully differentiated blood cell types, whereas the adult HSC lineage is arrested at the multipotent stem cell stage. One would also like to understand how the all-important property of self-renewal, that is the ability of the stem cell to divide without differentiating, is programmed. An understanding of these issues will have implications for the whole stem cell field.

Blood cells develop in close association with the endothelial cells that form the vasculature within which the blood cells circulate. In the case of the earliest wave of blood production, the yolk sac mesoderm expresses both blood and endothelial TFs and signal receptors, and later, these cells take the decision to make either blood or endothelium. These cells have therefore been referred to as hemangioblasts reflecting their potential to make both blood and endothelium (Kennedy et al., 2007). Because they give rise to the earliest blood cells only seen in the embryo, they have been called embryonic or primitive hemangioblasts. The mesoderm that gives rise to the DA and the HSCs is located in the lateral plate of the embryo proper (Fig. 1B) and also co-expresses both blood and endothelial TFs and signal receptors. However, in these cells, the expression of endothelial genes dominates (Fig. 1C), whereas in the primitive embryonic 
A

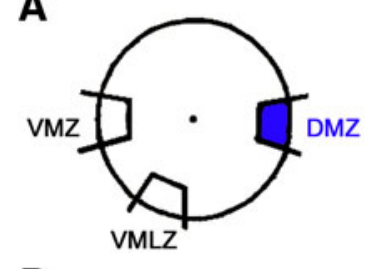

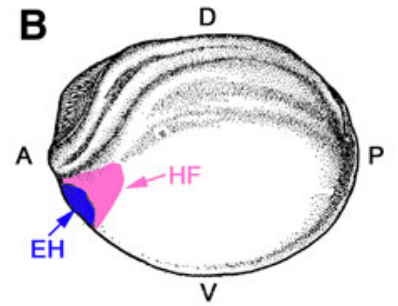
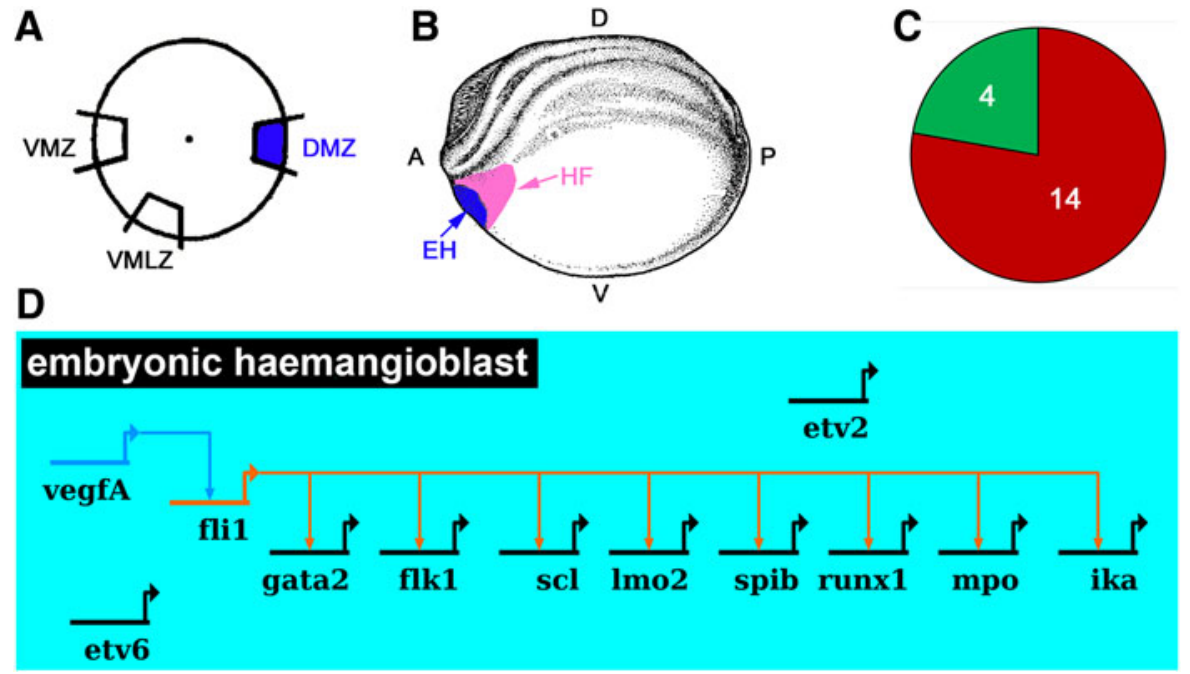

FIG. 2. Origins and programming of embryonic hemangioblast. (A) Embryonic hemangioblasts derive from the DMZ region of the gastrula embryo. (B) Embryonic hemangioblasts emerge in close association with the HF just after the end of gastrulation. (C) Pie chart illustrating the proportion of hematopoietic (red) and endothelial (green) gene expression in embryonic hemangioblasts. Numbers indicate the number of genes expressed in each category. (D) The embryonic hemangioblast gene regulatory network. Transcription factors critical for definitive hemangioblast such as Etv2 and Etv6 have no overt function in the specification of embryonic hemangioblasts. DMZ, dorsal marginal zone; HF, heart field.

hemangioblasts, the expression is dominated by blood regulators (Fig. 2C). This very likely reflects the differentiation decisions taken by the two distinct mesodermal populations, with the embryonic hemangioblast giving rise to mature blood cells as well as endothelium, and the definitive adult hemangioblast giving rise only to endothelium initially, namely the DA and posterior cardinal veins. However, a subset of this endothelium, namely the floor of the DA, goes on to give rise to the HSCs and has therefore been referred to as hemogenic endothelium.

The definitive adult hemangioblast expresses some key TFs associated with blood cell programming, such as Gata2, Scl, Lmo2, and Tel1/Etv6 (Ciau-Uitz et al., 2013a), but this expression is turned off while the cells migrate to the midline of the embryo to form the cord of endothelial cells making up the DA (Ciau-Uitz et al., 2000). The expression of these and the rest of the blood TFs comes back later in an ordered progression in the hemogenic endothelium. The function of the earlier expression in the definitive adult hemangioblast is unknown, but we assume that target blood genes are being prepared for activation. One possibility is that the chromatin of the blood genes to be expressed later is made accessible by a hit and run mechanism involving Gata2, Scl/Tal1, Lmo2, and Tel1/Etv6.

\section{THE DEFINITIVE ADULT HEMANGIOBLAST GENE REGULATORY NETWORK}

To better understand the mechanisms controlling the programming of the hemogenic endothelium in the DA, we have focused on its precursor population, the definitive adult hemangioblasts in the lateral plate mesoderm of the embryo. These are the cells co-expressing blood and endothelial markers, indicating that their potential has been restricted to these two outcomes, with the endothelial outcome chosen first. This cell population was first identified in the amphibian (Xenopus) model system, but evidence supports its conservation in mammalian organisms (Ciau-Uitz and Patient, 2016). We have profiled the gene expression of this cell population, with particular emphasis on TFs and signal receptors. In addition to the finding that the profile is dominated by endothelial genes (Fig. 1C), we have been able to uncover their regulatory relationships. To do this, we have combined temporal knowledge of expression patterns and the consequences of loss of function for each TF or signal receptor in turn. We have been able to 
build a gene regulatory network (GRN) for the definitive adult hemangioblast (Fig. 1D) (Ciau-Uitz et al., 2013a; Kirmizitas et al., 2017).

This GRN reveals many aspects of the programming of the definitive adult hemangioblast. First, it is clear that a key first step is to establish the expression of two TFs essential for the expression of the vascular endothelial growth factor (VEGF) receptor, Flk1/Kdr (Fig. 1D). The activation of this receptor by its ligand, vascular endothelial growth factor A (VEGFA), expressed initially in the adjacent somites, drives a gene expression cascade culminating in the expression of the hemangioblast specifier, Scl/Tall. However, the expression of this critical TF is also dependent on a VEGFA-independent gene cascade, which is triggered by the same two TFs that drive Flk1/Kdr expression. These two TFs are therefore critical drivers of the whole program. These two TFs are the E-Twenty-Six (ETS) factor, Fli1, and the blood-associated GATA factor, Gata2.

In view of the pivotal position of Fli 1 and Gata2 in the regulatory hierarchy establishing the definitive adult hemangioblast GRN, we set out to discover how their expression is triggered. A clue for Fli1 was fortuitously discovered while we were studying the role of miRNAs in blood development. By profiling the gene expression in the first hematopoietic cells emerging in the DA, we found that a particular miRNA was predominant. Furthermore, the gene locus for this miRNA is bound by many of the key hematopoietic TFs, and it is expressed in the definitive adult hemangioblasts (Nimmo et al., 2013). We therefore perturbed its expression and showed that it is required for Fli1 expression (Nimmo et al., 2013). Bioinformatic searching for likely targets for this miRNA revealed a receptor for transforming growth factor beta (TGF- $\beta$ ) signaling. Knockdown of this receptor enhanced expression of Fli1, whereas ectopic TGF- $\beta$ expression suppressed it. We therefore conclude that the expression of Fli1 in the lateral plate mesoderm is controlled by a doublenegative gate, with TGF- $\beta$ acting to restrict the domain of Fli1 expression (Fig. 1D). Double-negative gates are widely used in the embryo to restrict cell fates (Davidson and Levine, 2008).

The other critical TF at the top of the GRN hierarchy is Gata2. The expression of GATA factors is frequently under the control of bone morphogenetic protein (BMP) signaling. We therefore set out to perturb the BMP activity at various stages of development to determine if it had any input into Gata2 expression in definitive adult hemangioblasts. Somewhat to our surprise, we found multiple inputs into Gata2 expression but also a separate input into Flk1/Kdr expression (Fig. 1D) (Kirmizitas et al., 2017). BMP is therefore a critical regulator of blood stem cell development in the embryo.

VEGFA expression in the somites is clearly an early determinant of the definitive adult hemangioblast cell fate in the adjacent lateral plate mesoderm. We therefore set out to determine how this expression is controlled. We found that the blood ETS TF with leukemia connections, namely Etv6, previously known as Tel1, is the driving TF (Ciau-Uitz et al., 2010). Indeed, we found that Etv6 expression is induced in the lateral plate mesoderm as an early response to VEGFA signaling from the somites and that this Etv6 expression results in the expression of VEGFA within the lateral plate mesoderm, thus conferring cell autonomy to the developing definitive adult hemangioblasts after the initial signal from the somites (Fig. 1D). VEGFA has been shown to be required for self-renewal of the HSC (Gerber et al., 2002), offering a possible mechanistic link for Etv6/Tel1 in leukemia.

Significantly, our studies have shown that VEGFA plays multiple distinct roles in HSC programming, and it is, perhaps, the most important growth factor in this process. Three main isoforms of VEGFA (denominated as short, medium, and large) are generated by alternative splicing during development (Cleaver and Krieg, 1998). Critically, we have shown that these isoforms fulfill distinct functions in HSC programming: while the short isoform is sufficient for the generation of definitive hemangioblasts in the lateral plate, the formation of the DA and its arterialization, the medium/large isoforms are essential for hemogenic endothelium and HSC emergence (Leung et al., 2013). We have also demonstrated that another leukemic transcriptional repressor, Eto2, controls the generation of the medium/large isoforms and, by consequence, the emergence of HSCs (Leung et al., 2013).

In the literature, Etv6 had only been shown to act as a repressor (Hock and Shimamura, 2017). We were therefore interested to find out how it could be activating VEGFA expression. First, by profiling both the ETV6-dependent expression in somites and the binding of Etv6 to the genome, we were able to show that Etv6 binding is actually associated with both activation and repression ( $\mathrm{Li}$ et al., 2019). Second, we found that one of the repressed targets, FoxC3, turns out to be a repressor of VEGFA in in vitro experiments (Karadedou et al., 2012), so we tested the possibility that the repression of its expression by Etv6 is one way in which Etv6 positively regulates VEGFA and obtained supporting evidence. By sorting through the targets for positive regulation by Etv6, we found that one such target, Klf4, binds together with Etv6 to positively regulate VEGFA expression. Thus, Etv6 controls VEGFA expression by acting both positively and negatively. 


\section{THE PRIMITIVE EMBRYONIC HEMANGIOBLAST}

Our studies of the primitive embryonic hemangioblast are less advanced than those of the definitive adult hemangioblast. Nevertheless, we have shown that they develop in association with the heart field rather than the lateral plate (Fig. 2B) and that Fli1, Gata2, and VEGFA are also critical for the specification of these cells (Fig. 2D). However, VEGFA expression is not in this case driven by Etv6. Additionally, while Flk1/Kdr regulation is VEGFA-independent in the definitive adult hemangioblast, it is VEGFA-dependent in primitive embryonic hemangioblasts. Strikingly, depletion of the ETS factor, Etv2, which is required in the definitive adult hemangioblast, has no overt phenotype in primitive embryonic hemangioblasts, suggesting that myeloid-specific ETS TFs such as SpiB, which are absent in the definitive population, may compensate for its function. Thus, the expression and functions of the ETS family appear to be distinct but overlapping, raising the possibility that at least some of the different programming of the adult and embryonic populations is brought about by this family of TFs (Ciau-Uitz et al., 2013b).

The full biological consequences of all these differences are still to be appreciated mechanistically, but it is clear that the programming of these two related but importantly distinct cell populations can be distinguished.

\section{AUTHOR DISCLOSURE STATEMENT}

The authors declare there are no competing financial interests.

\section{REFERENCES}

Bertrand, J.Y., Chi, N.C., Santoso, B., et al. 2010. Haematopoietic stem cells derive directly from aortic endothelium during development. Nature 464, 108-111.

Boisset, J.C., van Cappellen, W., Andrieu-Soler, C., et al. 2010. In vivo imaging of haematopoietic cells emerging from the mouse aortic endothelium. Nature 464, 116-120.

Ciau-Uitz, A., and Patient, R. 2016. The embryonic origins and genetic programming of emerging haematopoietic stem cells. FEBS Lett. 590, 4002-4015.

Ciau-Uitz, A., Pinheiro, P., Gupta, R., et al. 2010. Tel1/ETV6 specifies blood stem cells through the agency of VEGF signaling. Dev. Cell 18, 569-578.

Ciau-Uitz, A., Pinheiro, P., Kirmizitas, A., et al. 2013a. VEGFA-dependent and -independent pathways synergise to drive Scl expression and initiate programming of the blood stem cell lineage in Xenopus. Development 140, 2632-2642.

Ciau-Uitz, A., Walmsley, M., and Patient, R. 2000. Distinct origins of adult and embryonic blood in Xenopus. Cell 102, 787-796.

Ciau-Uitz, A., Wang, L., Patient, R., et al. 2013b. ETS transcription factors in hematopoietic stem cell development. Blood Cells Mol. Dis. 51, 248-255.

Cleaver, O., and Krieg, P.A. 1998. VEGF mediates angioblast migration during development of the dorsal aorta in Xenopus. Development 125, 3905-3914.

Davidson, E.H., and Levine, M.S. 2008. Properties of developmental gene regulatory networks. Proc. Natl. Acad. Sci. U. S. A. 105, 20063-20066.

Gerber, H.P., Malik, A.K., Solar, G.P., et al. 2002. VEGF regulates haematopoietic stem cell survival by an internal autocrine loop mechanism. Nature 417, 954-958.

Hock, H., and Shimamura, A. 2017. ETV6 in hematopoiesis and leukemia predisposition. Semin. Hematol. 54, 98-104.

Ivanovs, A., Rybtsov, S., Ng, E.S., et al. 2017. Human haematopoietic stem cell development: From the embryo to the dish. Development 144, 2323-2337.

Karadedou, C.T., Gomes, A.R., Chen, J., et al. 2012. FOXO3a represses VEGF expression through FOXM1-dependent and -independent mechanisms in breast cancer. Oncogene 31, 1845-1858.

Kennedy, M., D'Souza, S.L., Lynch-Kattman, M., et al. 2007. Development of the hemangioblast defines the onset of hematopoiesis in human ES cell differentiation cultures. Blood 109, 2679-2687.

Kirmizitas, A., Meiklejohn, S., Ciau-Uitz, A., et al. 2017. Dissecting BMP signaling input into the gene regulatory networks driving specification of the blood stem cell lineage. Proc. Natl. Acad. Sci. U. S. A. 114, 5814-5821.

Kissa, K., and Herbomel, P. 2010. Blood stem cells emerge from aortic endothelium by a novel type of cell transition. Nature 464, 112-115. 
Leung, A., Ciau-Uitz, A., Pinheiro, P., et al. 2013. Uncoupling VEGFA functions in arteriogenesis and hematopoietic stem cell specification. Dev. Cell 24, 144-158.

Li, L., Rispoli, R., Patient, R., et al. 2019. Etv6 activates vegfa expression through positive and negative transcriptional regulatory networks in Xenopus embryos. Nat. Commun. 10, 1083.

Nimmo, R., Ciau-Uitz, A., Ruiz-Herguido, C., et al. 2013. MiR-142-3p controls the specification of definitive hemangioblasts during ontogeny. Dev. Cell 26, 237-249.

Yoder, M.C. 2014. Inducing definitive hematopoiesis in a dish. Nat. Biotechnol. 32, 539-541.

Address correspondence to:

Prof. Roger Patient

Weatherall Institute of Molecular Medicine

John Radcliffe Hospital

University of Oxford Oxford OX3 9DS

United Kingdom

E-mail: roger.patient@imm.ox.ac.uk 